\title{
Instruções Verbais e o Seguir Recomendações Médicas para Tratamento da Dermatite Atópica na Infância
}

\author{
Robson Zazula \\ Universidade Federal da Integração Latino-Americana, Foz do Iguaçu, Brasil \\ Márcia Cristina Caserta Gon* \\ Universidade Estadual de Londrina, Londrina, Brasil
}

\begin{abstract}
RESUMO
A dermatite atópica é uma doença crônica de pele com etiologia desconhecida e alta incidência na infância. O comportamento de seguir recomendações médicas é imprescindível para o controle da doença. Pode ser compreendido como um processo comportamental governado verbalmente e as instruções como importante variável. $\mathrm{O}$ estudo objetivou apresentar e discutir a instrução enquanto evento antecedente que controla o comportamento de seguir recomendações médicas por pacientes pediátricos. Este controle foi discutido nas interações entre as crianças e seus cuidadores, por meio da descrição de diferentes tipos de instruções e suas consequências. Foram apresentadas facilidades físicas e condições biológicas, o controle social e a história de reforçamento como variáveis que podem alterar o controle exercido pelas instruções.
\end{abstract}

Palavras-chave: dermatite atópica; instruções; tratamento médico; interação cuidador-criança.

\section{ABSTRACT \\ Verbal Instructions and the Follow of Medical Recommendations for Atopic Dermatitis Treatment in Childhood}

The atopic dermatitis is a skin chronic disease with unknown etiology and high incidence in childhood. Following medical recommendations is an indispensable behavior to the disease control. This behavior can be understood as a rule-governed behavior process and the instructions as an important variable. The aim of this study is to present and discuss the instruction, as an antecedent variable that controls the follow of recommendations' behavior by pediatric patients. This control was discussed in the interactions between children and their caregivers, through the description of different types of instructions and their consequences. Physical facilities and biological conditions, the social control and the role of reinforcement histories were described as variables that can change the control of instructions.

Keywords: atopic dermatitis; instructions; medical treatment; caregiver-child interaction.

No contexto da saúde, a ocorrência de inúmeras consequências não contingentes ao responder (i.e., apenas em longo prazo) torna o seguimento de recomendações médicas um processo comportamental, em grande parte, governado verbalmente. Neste sentido, as instruções verbais devem ser consideradas como importantes variáveis de controle (Buston \& Woods, 2000; Giarelli, Bernhardt, \& Pyeritz, 2010; Kyngås, 2000; Luciano \& Herruzo, 1992).
O seguimento de recomendações médicas pelo paciente e/ou seu cuidador de maneira correta envolve uma ampla gama de comportamentos em prol da saúde. Dentre os principais comportamentos, destacam-se aqueles relacionados diretamente ao tratamento (e.g., ingerir ou aplicar determinado medicamento, controlar a alimentação) ou que vão além, envolvendo mudanças no repertório total do indivíduo (e.g., identificar fatores orgânicos e/ou ambientais que podem provocar exacerbação dos sintomas) (Moraes, Rolim, \& Costa, 2009).

* Endereço para correspondência: Márcia Cristina Caserta Gon - marciagon@sercomtel.com.br 
Instruir é um comportamento observado nas interações sociais e culturais em situações cotidianas e, de modo mais complexo, em contextos específicos como o da saúde (Robles \& Gil, 2006). Por esta razão, o controle instrucional é imprescindível para a ampliação do repertório comportamental de autocuidado (Schimdt \& De Souza, 2003).

Culturalmente, estima-se que aproximadamente a partir dos sete anos de idade a criança comece a desempenhar um papel mais ativo quanto aos cuidados em relação à sua saúde de modo geral (Sanz, 2003). Sob esta perspectiva, quando a criança apresenta um problema de saúde, ela poderia realizar algumas tarefas relacionadas ao tratamento médico desde que supervisionada diretamente por um adulto. Estas tarefas são consideradas comportamentos de prevenção e de promoção à saúde, como utilizar alguns medicamentos (e.g., passar pomadas, segurar um comprimido na mão e ingeri-lo sem ajuda direta de seu cuidador). Além disso, ela já consegue entender o que é a sua doença, o que pode levar ao aparecimento e/ou piora dos sintomas e o que deverá fazer para evitar que ocorram tais consequências.

Portanto, quando se tratam de indivíduos que apresentam algum problema de saúde, agudo ou crônico, o autocuidado é considerado como uma classe operante que inclui comportamentos de tratamento da doença e que tem como principais funções controlar os sintomas, promover e manter uma boa condição de saúde. (Buston \& Wood, 2000; Giarelli et al., 2010; Kyngås, 2000). A apresentação deste repertório comportamental é importante no caso daqueles pacientes que recebem atendimento em contexto ambulatorial (i.e., que não necessitam de internação hospitalar) e que devem conduzir o tratamento em casa, como o que acontece com crianças que têm dermatite atópica, uma doença de pele de característica crônica.

A orientação para a realização do tratamento médico de pacientes com dermatite atópica ocorre primordialmente em contexto ambulatorial. Isto implica que o comportamento de seguir recomendações médicas seja imprescindível para o sucesso do tratamento, uma vez que estes não estão sob o cuidado e supervisão da equipe de saúde, como acontece com aqueles que estão internados (Greenlaw, Yentzer, O’Neill, Balkrishnan, \& Feldman, 2010). Assim, os cuidados médicos devem ser realizados exclusivamente por eles e/ou pelo seu cuidador em casa, no local de trabalho, de estudo ou de lazer.

O objetivo deste estudo é o de apresentar e discutir a instrução enquanto evento antecedente que controla (ou não) o comportamento de seguir recomendações médicas por pacientes pediátricos. Mais especificamente, esta discussão será focada na interação da criança com dermatite atópica e seu cuidador na ocasião de realizar o tratamento médico em casa. Inicialmente será apresentada uma breve introdução teórica sobre o controle instrucional e funções da instrução enquanto evento antecedente (i.e., estímulo discriminativo, operação estabelecedora e alteradora de função). Esta introdução tem por objetivo situar o leitor sobre o enfoque teórico segundo o qual a aplicação está sendo proposta e não o de fazer uma análise sistemática sobre o controle do comportamento por regras. Trata-se, portanto, de um ensaio sobre a aplicabilidade deste tipo de controle na área da saúde infantil.

Assim, será apresentada e discutida a instrução dada pelo cuidador para que a criança realize o tratamento, enfatizando-se a função discriminativa do estímulo verbal antecedente. A apresentação e a discussão serão ilustradas com instruções hipotéticas sobre tarefas de tratamento médico para dermatite atópica. De acordo com Valia (2008), estudos sobre o seguimento de recomendações médicas e o autocuidado com esta população são algo altamente promissor, dada a alta complexidade das classes de comportamento que devem ser emitidas (e.g., de passar pomada) e os baixos riscos à integridade física ao paciente. Além disso, é importante salientar que os conteúdos apresentados neste trabalho podem ser generalizados para outras situações, que envolvam pacientes pediátricos com doença crônica de pele como a psoríase e o vitiligo. A psoríase caracteriza-se pela ocorrência de lesões avermelhadas recobertas por escamas, localizadas no couro cabeludo, cotovelos e joelhos. Na infância pode acometer áreas como genitália, região glútea e periumbilical (Romiti, Maragno, Arnone, \& Takahashi, 2009). Por sua vez, o vitiligo caracteriza-se pelo surgimento de manchas brancas, localizadas ou disseminadas pelo corpo, resultantes da perda do pigmento natural da pele, a melanina (Silva, Gontijo, Pereira, \& Ribeiro, 2007). O tratamento médico destas doenças na infância, assim como na dermatite atópica, consiste do uso de emolientes e/ou umectantes, asso- 
ciados a corticosteroides tópicos, fototerapia e, nos casos mais graves, podem requerer terapia sistêmica com imunossupressores (Romiti et al., 2009; Silva et al., 2007).

\section{O CONTROLE INSTRUCIONAL}

O controle instrucional, segundo Catania (1998), pode ser compreendido como a relação entre o comportamento emitido verbalmente pelo falante (antecedente verbal) e a contingência descrita na instrução sob a qual o ouvinte deverá se comportar. Por estar sob controle de consequências descritas pelo estímulo discriminativo verbal e não pelo contato direto com elas, a emissão do comportamento do ouvinte ocorre independentemente de suas consequências naturais (Schimdt \& De Souza, 2003; Skinner, 1969).

Instruções, segundo Paracampo e Albuquerque (2005), podem ser definidas como estímulos verbais antecedentes que especificam contingências, assim como avisos, orientações, conselhos e ordens. Elas descrevem o comportamento a ser emitido, as condições sob as quais deve ocorrer e suas possíveis consequências. Além disso, outros autores (Braam \& Malott, 1990; Reitman \& Gross, 1996; Schlinger, 1990) definem instruções como sendo aquelas que descrevem as três partes da tríplice contingência, que pode ser realizada de modo completo (i.e., antecedente, resposta e consequente) ou de modo incompleto (i.e., antecedente e resposta, resposta e consequência, antecedente e consequência).

De acordo com Albuquerque (1991, 2001), regras podem exercer múltiplas funções. Assim como contingências de reforço que elas descrevem, as regras podem produzir uma restrição de variabilidade comportamental, estabelecer comportamentos novos e alterar funções de estímulos (Albuquerque, 2001; Paracampo \& Albuquerque, 2005). Na sequência, serão apresentadas brevemente as três funções da regra ou das instruções.

A primeira função que pode ser exercida pelas instruções é a de operações estabelecedoras (ou operações motivacionais). Neste caso, as instruções podem alterar momentaneamente a efetividade reforçadora de determinado estímulo e evocar classes de respostas que foram reforçadas no passado pelo mesmo estímulo (Michael, 1982, 1993).
A instrução pode ter a função de alterador de função. De acordo com Blakely e Schillinger (1987), isto ocorre porque os efeitos das instruções são atrasados. Nestes casos, os efeitos evocativos dos estímulos se tornam mais aparentes na medida em que aumenta o intervalo de tempo entre a apresentação da instrução e o surgimento do estímulo, cuja função foi alterada pela regra.

Por fim, a instrução pode ter a função de estímulo discriminativo. De acordo com Skinner (1966, 1969), as instruções podem ser compreendidas como estímulos especificadores de contingências, sendo parte integrante de um conjunto de contingências de reforço. Neste caso, as instruções podem descrever a relação entre eventos que antecedem a resposta, a própria resposta e suas possíveis consequências. Dentre os possíveis exemplos, podem-se destacar os avisos, as orientações, as recomendações e as ordens, tal como será apresentado ao longo do presente estudo.

\section{Dermatite Atópica: Instruções sobre a condução do tratamento médico pelos cuidadores à criança}

Devido ao curso crônico da dermatite atópica, recomendações médicas devem ser seguidas pelo paciente, visando minimizar a ocorrência dos sintomas e o agravamento da doença. Além da cronicidade, o tratamento é altamente complexo, envolvendo várias recomendações e a emissão de uma ampla classe de respostas pelo paciente (Valia, 2008). Dentre estas se destacam: a) evitar contato com substâncias irritantes (e.g., sabão e produtos de limpeza, cosméticos, roupas de lã ou sintéticas), b) evitar contato com substâncias aeroalergênicas (e.g., poeira, pelo de animais, perfumes ou fumaça), c) hidratação da pele, d) usar adequadamente medicamentos tópicos e/ou orais; e) evitar coçar ou irritar a pele e f) manter as unhas curtas para evitar escoriações (Kienast \& Hoeger, 2010; Sampaio \& Rivitti, 1998). Uma vez prescritas, o médico espera que o paciente siga tais recomendações para que o tratamento seja bem-sucedido.

Entretanto, apesar de haver uma relação diretamente proporcional entre maiores índices no seguimento das recomendações médicas e consequente melhora dos sintomas, observa-se que entre $30 \%$ e $50 \%$ dos pacientes com dermatite atópica não realizam o tratamento indicado pelo médico (Krejci-Manwaring et al., 2006; Serup et al., 2006). Dentre todas as queixas, 
uma das mais frequentes, especialmente dos cuidadores, é o não seguimento das recomendações médicas pelos pacientes (Lawson, Lewis-Jones, Finlay, Reid, \& Owens, 1998).

Um dos fatores mais importantes para que haja maiores índices de seguimento de recomendações médicas é uma adequada descrição das contingências relacionadas ao tratamento médico, realizada frequentemente pelos pais e/ou cuidadores dos pacientes. Assim, a análise da instrução sobre o tratamento médico, verbalizada pelo cuidador à criança com a doença, é fundamental para se compreender o seu controle na emissão de comportamentos de segui-la. Além disso, as instruções de cuidadores que especifiquem adequadamente contingências e classes comportamentais de autocuidado a serem emitidas pelo paciente pediátrico podem aumentar a frequência de comportamentos relacionados à adesão ao tratamento médico no futuro, desempenhando um importante papel no desenvolvimento da autonomia da criança e do adolescente em relação a estes cuidados (McMahon \& Forehand, 2005).

Observa-se que a comunicação, entendida como a descrição de contingências relacionadas ao tratamento médico, é uma das principais dificuldades enfrentadas pelos cuidadores ao instruir crianças quanto ao seguimento de recomendações médicas e, consequentemente, na aquisição e manutenção de repertórios comportamentais de autocuidado (e.g., Oliveira \& Gomes, 2004; Zolnierek \& DiMatteo, 2009). Embora existam evidências empíricas sobre o controle exercido por regras no comportamento de segui-las (e.g., Braam \& Mallot, 1990; Harding et al., 1994; Hupp \& Reitman, 1999; Paracampo \& Albuquerque, 2005; Albuquerque \& Paracampo, 2010; Reitman \& Gross, 1996; Roberts, McMahon, Forehand, \& Humphreys, 1978; Robles \& Gil, 2006), poucos estudos são conduzidos na área da saúde. Deste modo, na próxima seção serão apresentados exemplos hipotéticos de situações de tratamento médico na interação entre a criança com dermatite atópica e seu cuidador, relacionando-as aos diferentes tipos de instrução.

\section{Instrução enquanto estímulo discriminativo: apresentação e exemplos em dermatite atópica}

As prescrições médicas são, em alguns casos, compostas por instruções simples a serem seguidas repetidamente por curtos ou longos períodos de tempo e/ou em outros casos, compostas por uma série complexa de instruções a serem cumpridas pelo paciente e/ou seu cuidador em uma sequência específica. Elas podem conter informações simples ou complexas sobre os comportamentos que devem ser emitidos e possíveis reforçadores contingentes a esta emissão (Baron \& Galizio, 1983; Bentall \& Lowe, 1987; Luciano \& Herruzo, 1992; Roberts et al., 1978).

\section{Instruções simples e complexas}

Instruções simples envolveriam descrições de contingências comportamentais com baixa complexidade e que deveriam ser realizadas repetidamente ao longo de um determinado período de tempo (Luciano \& Herruzo, 1992). Um exemplo hipotético seria o cuidador instruir a criança com dermatite atópica para aplicar o medicamento na pele da seguinte forma: "Você deve passar a pomada que o médico receitou nas partes de seu corpo que estão coçando ou que estão irritadas e avermelhadas. Isso diminuirá a coceira".

Séries complexas de instruções envolvem, por sua vez, extensas cadeias comportamentais que devem ser emitidas em determinadas condições e/ou sequência (Luciano \& Heruzzo, 1992). No caso de pacientes pediátricos com dermatite atópica, um exemplo seria o cuidador instruir a criança da seguinte forma: “a) logo ao sair do chuveiro, b) você deve apenas enxugar-se com a toalha sem esfregá-la na pele e c) espalhar bastante hidratante em todas as partes de seu corpo, ficando 'bem melecado', porque isso diminuirá o ressecamento de sua pele e ajuda a diminuir as coceiras".

Luciano e Heruzzo (1992) enfatizam ainda que, para aquelas crianças e adolescentes que apresentam repertórios comportamentais verbais e não verbais para executar tarefas relacionadas ao tratamento médico, a verbalização de instruções acerca do comportamento-alvo deve ser sistemática, em especial, nas primeiras fases do tratamento. A verbalização dessas instruções em partes, de modo sistemático e contínuo, como apresentada no exemplo acima, pode diminuir o grau de complexidade da tarefa e aumentando, assim, a probabilidade em seguir as recomendações. Outro fator destacado pelos autores, que justificaria a repetição sistemática das instruções do cuidador para a criança, é que algumas prescrições, por envolverem cadeias complexas de comportamentos, podem ser difí- 
ceis de serem seguidas. Portanto, instruções verbalizadas em partes, com uma sequência específica e de modo sistemático, aumentam a probabilidade de a criança apresentar comportamentos de autocuidado.

\section{Instruções completas}

É importante afirmar que uma instrução simples não deve ser limitada, (i.e., ao envolver a descrição de poucos elementos da contingência comportamental, $o$ que restringe a emissão de comportamentos pelo ouvinte). Isso porque, conforme discutem Baron e Galizio (1983), quanto mais limitadas forem as instruções verbalizadas pelo falante, menor será o controle exercido por elas sobre o comportamento do ouvinte. Além disso, Bentall e Lowe (1987) constataram que, mesmo em crianças de diferentes faixas etárias, quanto mais específica e completa for a instrução, maior a probabilidade de que elas sigam as recomendações e mais precisa será a resposta.

A discussão de Baron e Galizio (1983) e Bentall e Lowe (1987) também é sustentada por Roberts et al. (1978), Lowe (1979), Bramm e Mallot (1990), Reitman e Gross (1996) e, mais recentemente, por Hupp et al. (2008). Estes pesquisadores afirmaram que a forma como a instrução é verbalizada para a criança pode influenciar no seguimento ou não das recomendações, a serem seguidas para a aplicação do medicamento. Instruções apresentadas de modo claro, simples e direto são mais prováveis de serem seguidas do que as genéricas, ambíguas ou hesitantes. Como exemplos hipotéticos dessas, pode-se citar: "vá até o seu quarto, pegue o tubo de pomada na primeira gaveta da mesinha ao lado da cama e traga para mim".

Segundo Harding et al. (1994) e Bouxsein et al. (2008), instruções genéricas, ambíguas ou hesitantes podem ser compreendidas como aquelas cujo direcionamento do indivíduo para a emissão de comportamentos ocorre de modo não específico ou pouco claro. Além disso, apresentam menor probabilidade de seguimento, podendo levar o indivíduo a responder de forma errática e variável (Lowe, 1979). No caso da dermatite atópica, a verbalização de instruções vagas ou pouco claras pode dificultar a realização de determinados procedimentos médicos pela criança (e.g., descrição imprecisa e não direcionada à criança acerca de como aplicar medicamentos tópicos, descrever genericamente condições ambientais que aumentam a probabilidade de exacerbação dos sintomas, quais classes de respostas emitir, etc.). Como exemplo, pode-se citar uma situação hipotética na qual a pele começa a ficar avermelhada e a criança se coça. A mãe, ao ver o que está acontecendo, verbaliza a seguinte instrução: "Precisa cuidar".

De acordo com Braam e Mallot (1990), uma descrição, para ser caracterizada como completa, deve especificar quais os comportamentos que o indivíduo deve emitir, em qual contexto deve ocorrer e quais são as consequências efetivas daquele responder. Este fato não é observado no exemplo hipotético mencionado no parágrafo anterior. Assim, em se tratando de pacientes pediátricos com dermatite atópica, é importante que as instruções sejam completas e especifiquem qual medicamento deverá ser aplicado, qual o intervalo entre as aplicações, como aplicá-lo e quais as consequências efetivas desse tratamento, como: "é importante que você passe o creme hidratante todos os dias após o banho, para que sua pele não fique seca e ardendo".

De acordo com Luciano e Herruzo (1992), além das instruções verbais (i.e., recomendações médicas), outro componente essencial no processo de adesão ao tratamento é a descrição das consequências da emissão (ou não) dos comportamentos relacionados ao tratamento médico. Tal descrição é importante para aumentar a probabilidade de emissão desses comportamentos, devido ao fato de que as consequências deste ocorrem apenas em longo prazo. Entretanto, outras condições são igualmente importantes no processo de adesão ao tratamento, além do tipo de instrução e da descrição da consequência. Deste modo, estas condições, bem como as consequências do comportamento de seguir recomendações médicas, serão apresentadas como importantes componentes do comportamento de seguir recomendações médicas.

\section{Seguimento de Instruções ao tratamento médico da dermatite atópica}

As consequências de emitir comportamentos descritos na instrução podem ser de ação direta ou imediata, com alta probabilidade de ocorrência e efeito significativo sobre o responder do indivíduo, e de ação indireta ou atrasada, que apresentam baixa probabilidade de ocorrência, efeito pouco significativo sobre o responder ou cumulativo ao longo do tempo (Braam \& Mallot, 1990; Luciano \& Herruzo, 1992). 
Em relação ao tratamento médico de crianças com dermatite atópica, são exemplos de consequências imediatas que agem diretamente sobre o organismo: as consequências do seguir a regra e entrar em contato com a diminuição dos sintomas aversivos da doença (e.g., diminuição da coceira, ressecamento), o aumento da probabilidade de piora dos sintomas, caso a criança não aplique a pomada nas lesões (e.g., dor, a irritação da pele e coceira). Paracampo et al. (2001) afirmam que o comportamento se seguir regras pode estar diretamente relacionado ao tipo de consequências. Com relação às consequências imediatas, por exemplo, supõe-se que há maior probabilidade de seguimento da instrução para aplicar o medicamento pela criança quando há elevada possibilidade para minimizar a ocorrência de consequências aversivas (i.e., irritação e prurido da pele).

Quanto à descrição de consequências atrasadas, um exemplo seria a probabilidade de complicações no quadro clínico da criança por não seguir as recomendações para o tratamento. Neste caso, pode-se afirmar que algumas recomendações médicas podem descrever comportamentos, cujas consequências ocorrem apenas em longo prazo e não afetam diretamente o comportamento da criança de segui-las ou ocorrem apenas após os efeitos cumulativos da emissão de uma série de comportamentos de cuidados com a saúde. De acordo com Matos (2001), quando situações em que consequências naturais de um comportamento são fracas, têm magnitude pequena ou operam em longo prazo, o emprego de regras é especialmente importante. São nestas situações que as consequências mais arbitrárias, mediadas socialmente, passariam a exercer controle sobre o comportamento de seguir instruções. De acordo com Zettle e Hayes (1982) o comportamento de seguir a regra seria controlado por duas fontes de variáveis que descrevem contingências, mas que são distintas entre si: o pliance e o tracking.

O pliance é classificado como um comportamento de seguir regras. É emitido devido à história de consequências mediadas socialmente para uma correspondência formal entre o estímulo verbal antecedente (e.g., instrução) e o comportamento-alvo relevante (e.g., obediência), ou seja, o comportamento de seguir regras pelo ouvinte seria controlado por consequências mais arbitrárias (Hayes, Zettle, \& Rosenfarb, 1989; Schlinger \& Blakely, 1987). Suponha que a mãe diga à criança: "Passe a pomada e a coceira vai melhorar". Passar a pomada é um pliance se a criança o fizer em virtude de uma história de consequências mediadas socialmente por seguir a regra, como por exemplo, a mãe ter reforçado positivamente o comportamento de fazê-lo.

O tracking é também classificado como um comportamento de seguir regra, porém é apresentado devido à história de correspondência formal entre o estímulo verbal antecedente e o contato direto com a consequência que segue a sua emissão (Hayes, Zettle, \& Rosenfarb, 1989; Schlinger \& Blakely, 1987). Por exemplo, se a criança passa a pomada e a coceira diminui, o comportamento de seguir a regra estaria sendo mantido pelas suas consequências naturais.

Contudo, observa-se por meio destes exemplos citados nos parágrafos anteriores que não é a topografia do comportamento de seguir a regra que o classifica como sendo de pliance ou de tracking, mas as variáveis que o controlam (Hayes et al., 1989; Schlinger \& Blakely, 1987). Se a criança segue a regra devido ao controle da comunidade verbal que pune o não seguir a regra e reforça o segui-la, isto é um comportamento de pliance. Por outro lado, se ela segue a regra devido às consequências naturais produzidas pelo seu seguimento, isto é um comportamento de tracking (Paracampo \& Albuqurque, 2005).

Embora a descrição das recomendações médicas e das consequências para seu seguimento seja importante no controle do comportamento de realizar o tratamento, não se pode garantir que as regras sejam seguidas pelo paciente. Isto porque apenas a verbalização de uma instrução de modo completo ou incompleto pode não ser suficiente para produzir mudanças no comportamento do ouvinte (Baum, 2006; Sério, 2004). Ao se compreender a instrução como um estímulo verbal antecedente, deve-se analisar a relação existente entre a instrução e o comportamento do ouvinte de segui-la, para se determinar as variáveis ambientais que podem afetar seu responder (Schutte \& Hopkins, 1970). Contudo, analisar o comportamento do ouvinte de seguir instruções, mais especificamente de crianças e adolescentes com dermatite atópica, conduz, na área da saúde infantil, a uma discussão sobre obediência contingente às instruções do cuidador de realizar o tratamento médico, além da análise do controle por regras per se. Deste modo, para fins 
da análise, a obediência será apresentada na seção seguinte como um comportamento operante, modelado ao longo da história de vida do indivíduo, e que é controlado por contingências que envolvem operações de consequenciação como propõem Bentall e Lowe (1987).

\section{Obediência Infantil às Instruções de Tratamento Médico pelo Cuidador: Faixa etária, facilidades físicas, condições biológicas da criança e operações motivacionais}

Segundo Wahler (1997), obediência é um termo usado para descrever o comportamento do indivíduo em seguir instruções, ordens ou pedidos. No contexto da saúde, especialmente na pediatria, o termo também pode ser compreendido como a realização de tarefas relacionadas ao tratamento, a partir das recomendações médicas (i.e., instruções verbais). Estas podem ser verbalizadas pelos cuidadores que acompanham o tratamento de suas crianças no dia a dia, envolvendo, neste sentido, o seguimento de recomendações médicas (Arruda \& Zannon, 2002).

Sendo a obediência um comportamento operante, este pode ser modelado e fortalecido. Dentre as variáveis que influenciam na sua modelagem, pode-se citar o repertório verbal apresentado pela criança, o comportamento daquele que a instrui, as instruções verbais enquanto estímulos antecedentes, bem como as consequências para o comportamento de seguir tais instruções. Embora o comportamento seja especificado pela instrução, o responder ocorre apenas quando a ocasião para a obediência for estabelecida (Cerutti, 1989), tornando-se importante a compreensão das demais variáveis como faixa etária, facilidades físicas, condições biológicas da criança e operações motivacionais como parte da interação cuidador e criança.

\section{Faixa etária da criança}

As classes de respostas consideradas obedientes ou desobedientes podem variar de acordo com a faixa etária, segundo os repertórios comportamentais apresentados pela criança (Bentall \& Lowe, 1987). No caso de pacientes pediátricos mais novos (e.g., idade pré-escolar), observa-se que a obediência em relação às tarefas do tratamento ocorre de modo unidirecional, com maior participação dos cuidadores. Com estas, é maior a probabilidade de o cuidador instruir a criança de modo simples e direto para realizar uma tarefa específica, e ao mesmo tempo orientá-la fisicamente para fazê-la. Com crianças em idade escolar e adolescentes é esperado que o uso de orientação física ${ }^{1}$ pelo cuidador diminua e o comportamento considerado obediente fique mais diretamente sob o controle das solicitações para realização do tratamento. Pesquisas demonstram que o comportamento de obediência de crianças em idade escolar e adolescentes ficam sob maior controle de instruções verbais do que o de crianças mais novas (Bentall \& Lowe, 1987; Bentall, Lowe \& Beasty, 1985; Blum, Willians, Friman, \& Christophesen, 1995). Assim, crianças em idade pré-escolar com dermatite atópica necessitariam ainda ser orientadas fisicamente na realização do tratamento médico, enquanto as mais velhas ou adolescentes poderiam realizá-lo de modo autônomo, sem que o cuidador as orientasse fisicamente.

\section{Facilidades físicas e condições biológicas da criança}

Entretanto, outras variáveis, além da idade da criança, devem ser consideradas ao se avaliar a aquisição e manutenção do comportamento de obediência infantil às instruções do cuidador para realizar tratamento médico. De acordo com Paracampo e Albuquerque (2005), o seguimento de instruções depende mais da combinação de uma série de condições favoráveis do que de uma condição ou outra isoladamente. Dentre essas, destacam-se as facilidades físicas e condições biológicas do paciente para emissão de comportamentos descritos na instrução e que são indispensáveis para que ocorra a obediência. A febre, a coceira, o medicamento ou uma simples colher de chá para medi-lo poderão controlar diretamente o comportamento especificado pela regra (Luciano \& Herruzo, 1992). Por exemplo, se não há um hidratante em casa, a regra "Passe hidratante diariamente após o banho" não poderá ser seguida.

\section{Operação motivacional como parte da interação cuidador e criança e o seguimento de instruções para a realização do tratamento médico}

É importante destacar também o papel desempenhado pela história de reforçamento do indivíduo. Isso porque a história determina o comportamento atual que está sob o controle da instrução. Como citado em 
parágrafos anteriores, as instruções poderiam ser seguidas porque o comportamento de segui-las no passado, em situações semelhantes às atuais, evitou punição social ou foi reforçado positiva ou negativamente (Paracampo, \& Albuquerque, 2005). Neste contexto, o papel desempenhado pelo indivíduo que dá a instrução para realizar as prescrições é extremamente importante para o seu seguimento (Luciano \& Herruzo, 1992; Masur \& Anderson, 1988). Para crianças e adolescentes, quanto mais positivas são as interações com seu cuidador (e.g., mais reforço positivo menos coerção e punição física e verbal contingente aos comportamentos de autocuidado), maior a probabilidade de seguimento das solicitações, bem como a modelagem e manutenção de repertórios comportamentais relacionados ao tratamento médico.

Portanto, pode-se hipotetizar que as crianças com dermatite atópica, cujos cuidadores estabelecem interações mais positivas com seus filhos, apresentarão maior probabilidade de emitir comportamentos relacionados ao tratamento do que aquelas cujos cuidadores estabeleceram interações aversivas ou punitivas e maior probabilidade de autonomia em relação aos cuidados com a saúde no futuro.

Como exemplos hipotéticos de interações positivas, podem-se citar uma mãe que conta histórias divertidas ao seu filho no momento em corta suas unhas ou uma mãe que após o banho permite ao seu filho brincar de passar creme, se "lambuzar" ou fazendo desenhos com os eles na pele. No primeiro exemplo, o comportamento da mãe de contar histórias poderia ser uma operação estabelecedora (Michael, 1993, 2000) por alterar o valor das consequências reforçadoras (i.e., saúde da pele) e as probabilidades de comportamentos reforçados por elas (i.e., cuidar da pele). De acordo com Menezes e Gon (2012), a manipulação de variáveis antecedentes como "atenção parental" pode ser uma alternativa eficaz para aumentar a probabilidade de ocorrência de respostas de obediência às instruções para realizar o tratamento médico e minimizar o uso de consequências aversivas pelo cuidador em interações com a criança. No segundo exemplo, a mãe poderia elogiar descritivamente a criança em relação à emissão de comportamentos de autocuidado (passar o creme na pele), mesmo que esta não esteja fazendo-o de forma correta. Aqui, a consequenciação, por meio de elogios que descrevem o comportamento da crian- ça, como por exemplo, "ótimo que você espalhou o creme pela pele!" e não, "pare com isso, você está se melecando todo e estragando tudo!", poderia aumentar a probabilidade de que criança passe o creme na pele após o banho, independentemente da topografia do comportamento apresentada.

\section{CONSIDERAÇÕES FINAIS}

Ao se avaliar as instruções verbais enquanto estímulos discriminativos para o comportamento de seguir instruções, um amplo conjunto de condições deve ser analisado, tais como o repertório verbal daquele que segue a instrução, o comportamento do falante, as instruções enquanto estímulos discriminativos para o ouvinte, bem como as consequências do comportamento se seguir a instrução.

Conhecer tais condições é fundamental para a atuação do analista do comportamento em diferentes campos do conhecimento, em especial o da saúde. Nesta área, tal conceito pode ser aplicado em questões relacionadas ao seguimento das recomendações médicas e adesão ao tratamento. De acordo com Luciano e Herruzo (1992), o seguimento de prescrições médicas pode ser compreendido como comportamento governado verbalmente, uma vez que envolvem a descrição, pelo falante de consequências em longo prazo, relacionadas aos cuidados à saúde. A emissão, pelo falante, deste comportamento é fundamental no processo de adesão ao tratamento e está diretamente relacionado à aprendizagem de comportamentos de autocuidado que devem ser mantidos por toda a vida do indivíduo.

Neste sentido, sugere-se, como propostas de estudos futuros, a realização de um maior número de pesquisas empíricas e teóricas acerca das variáveis que influenciam o seguimento de recomendações médicas por pacientes pediátricos com dermatite atópica. É importante que estas levem em consideração as variáveis que possam influenciar no seguimento das recomendações pelos pacientes. Deste modo, possibilita-se o desenvolvimento de estratégias de intervenção direcionadas a esta população e a outras que apresentam também algum problema de saúde crônico e que necessitam continuar o tratamento médico fora do ambiente hospitalar. 


\section{REFERÊNCIAS}

Albuquerque, L. C. (1991). Efeitos de regras no controle do comportamento humano. [Resumo]. In. Sociedade Brasileira de Psicologia (Org.), Resumos de comunicações científicas (p.162). Ribeirão Preto: SBP.

Albuquerque, L. C. (2001). Definições de regras. In. H. J. Guilhardi,M. B. B. P. Madi, P. P. Queiroz, P. P., \& M. C. Scoz (Orgs.). Sobre comportamento e cognição: Expondo a variabilidade (pp. 132-140). Santo André: ARBytes.

Albuquerque, L. C., \& Paracampo, C. C. P. (2010). Análise do controle por regras. Psicologia USP, 21(2), 253-273.

Arruda, P. M. A., \& Zannon, C. M. L. C. (2002). Adesão ao tratamento pediátrico da doença crônica evidenciando o desafio enfrentado pelo cuidador. In C. M. L. C. Zannon (Org.), Tecnologia Comportamental em Saúde (pp. 113). Santo André: ESETec.

Baron, A., \& Galizio, M. (1983). Instructional control of human operant behavior. The Psychological Record, 33, 495-520.

Baum, W. M. (2006). Compreender o Behaviorismo: Comportamento, cultura e evolução ( $2^{\mathrm{a}}$ ed.) Porto Alegre: Artmed.

Bentall, R. P., \& Lowe, C. F. (1987). The role of verbal behavior in human learning: III. Instructional effects in children. Journal of Experimental Analysis of Behavior, 47(2), 177-190.

Bentall, R. P., Lowe, C. F., \& Beasty, A. (1985). The role of verbal behavior in human learning: II. Developmental differences. Journal of the Experimental Analysis of Behavior, 43, 165-181.

Blakely, E., \& Schillinger, H. (1987). Rules: Functionaltering contingency-specifying stimuli. The Behavior Analyst, 10, 183-187.

Blum, N. J., Willians, G. E., Friman, P. C., \& Christophersen, E. R. (1995). Disciplining Young Children: The Role of Verbal Instructions and Reasoning. Pediatrics, 2(1), 336-341.

Bouxsein, K. J., Tiger, J. H., \& Fisher, W. W. (2008). A comparison of general and specific instructions to promote task engagement and completion by a young man with asperger syndrome. Journal of Applied Behavior Analysis, 41(1), 113-116.

Braam, C., \& Malott, R. W. (1990). "I'll do it when the snow melts": the effects of deadlines and delayed outcomes on rules-governed behavior in preschool children. The Analysis of Verbal Behavior, 8, 67-76.

Buston, K. M., \& Wood, S. F. (2000). Non-compliance amongst adolescents with asthma: listening to what they tell us about self-management. Family Practice, 17(2), 134-138.

Buston, K. M., \& Wood, S. F. (2000). Non-compliance amongst adolescents with asthma: listening to what they tell us about self-management. Family Practice, 17(2), 134-138.

Carvalho, J. F. (2012). Programa de orientação comportamental em grupo para pais de crianças com dermatite atópica (Dissertação de Mestrado). Universidade Estadual de Londrina, Londrina.

Catania, A. C. (1998). Learning (4 ${ }^{\text {th }}$ ed.). New Jersey: Prentice Hall.

Cerutti, D. T. (1989). Discrimination theory of rulegoverned behavior. Journal of the Experimental Analysis of Behavior, 51, 259-276.

Giarelli, E., Bernhardt, B. A., \& Pyeritz, R. E. (2010). Selfsurveillance by adolescents and young adults transitionning to self-management of a chronic genetic disorder. Health Education \& Behavior, 37(1), 133-150.

Greenlaw, S. M., Yentzer, B. A., O’Neill, J. L., Balkrishnan, R., \& Feldman, S. R. (2010). Assessing adherence to dermatology treatments: a review of self-report and electronic measures. Skin Research and Technology, 16(2), 253-258.

Hanna, K. M., \& Guthrie, D. (2001). Parents' and adolescents' perceptions of helpful and no helpful support for adolescents' assumption of diabetes management responsibility. Issues in Comprehensive Pediatric Nursing, 24, 209-223.

Harding, J., Wacker, D. P., Cooper, L. J., Millard, T., \& Jensen-Kovalan, P. (1994). Brief hierarquical assessment of potential treatment components with children in an outpatient clinic. Journal of Applied Behavior Analysis, 27(2), 291-300.

Hayes, S. C., Zettle, R. D., \& Rosenfarb, I. (1989). Rule following. In S. C. Hayes (Ed.), Rule-governed behavior: Cognition, contingencies, and instructional control (pp. 191-220). New York: Plenum.

Hupp, S. D., \& Reitman, D. (1999). The effects of stating contingency-specifying stimuli on compliance in children. The Analysis of Verbal Behavior, 16, 17-27.

Hupp, S. D., Reitman, D., Forde, D. A., Schiver, M. D., \& Kelley, M. L. (2008). Advancing the assessment of parent-child interaction: development of the Parent Instruction-Giving Game with the Youngster. Behavior Therapy, 39, 91-106.

Kienast, A. K., \& Hoeger, P. H. (2010). Atopic dermatitis in children: what to do when nothing works. Giornale Italiano di Dermatologia e Venereologia, 145(2), 303308.

Krejci-Manwaring, J., McCarty, M. A., Camacho, F., Carroll, C. L., Johnson,K., Manuel, J., et al (2006). Adherence with topical treatment is poor compared with adherence with oral agents: implications for effective clinical use of topical agents. Journal of America Academy of Dermatology, 54(5 Sup), 235-236.

Kyngas, H. (2000). Compliance of adolescents with chronic disease. Journal of Clinical Nursing, 9, 549-556. 
Lawson, V., Lewis-Jones, M., Finlay, A., Reid, P., \& Owens, R. (1998). The family impact of childhood atopic dermatitis: the Dermatitis Family Impact questionnaire. British Journal of Dermatology, 138(1), 107-113.

Lowe, C. F. (1979). Determinants of human operant behavior. In M. D. Zeiler, \& P. Harzem (Eds.), Advances in Analysis of Behavior: Vol. 1. Reinforcement and the Organization of Behavior (pp. 159-192). Chichester, England: Wiley.

Luciano, M. C., \& Herruzo, J. (1992). Some relevants components of adherence behavior. Journal of Behavior Therapy and Experimental Psychiatry, 23(2), 117-124.

Masur, F. T., \& Anderson, K. (1988). Adhesión del paciente al tratamiento: un reto para la psicología de la salud. Revista Latinoamericana de Psicología, 20(1), 103-126.

Matos, M. A. (2001). Comportamento governado por regras. Revista Brasileira de Terapia Cognitiva e Comportamental, 3(2), 51-66

McMahon, R. J., \& Forehand, R. L. (2005). Helping the Noncompliance Child: Family-Based Treatment for Oppositional Behavior. New York: Guilford Press.

Menezes, C. C., \& Gon, M. C. C. (2012). A Influência de Eventos Antecedentes nos Problemas de Comportamento Infantil: Revisão de Conceitos e Aplicabilidade. Interação em Psicologia, 15(2), 169-177.

Michael, J. (1982). Distinguishing Between Discriminative and Motivational Functions of Stimuli. Journal of Experimental Analysis of Behavior, 37, 149-155.

Michael, J. (1993). Establishing operations. The Behavior Analyst, 16(2), 191-206.

Michael, J. (2000). Implications and refinements of the establishing operation concept. Journal of Applied Behavior Analysis, 33, 401-410.

Moraes, A. B. A., Rolim, G. S., \& Costa, A. (2009). O processo de adesão numa perspectiva analíticocomportamental. Revista Brasileira de Terapia Comportamental e Cognitiva, 11(2), 329-345.

Oliveira, V. Z., \& Gomes, W. B. (2004). Comunicação médico-paciente e adesão ao tratamento em adolescentes portadores de doenças orgânicas crônicas. Estudos de Psicologia (Natal), 9(3), 459-469.

Paracampo, C. C. P., \& Albuquerque, L. C. (2005). Comportamento controlado por regras: revisão crítica de proposições conceituais e resultados experimentais. Interação em Psicologia, 9(2), 227-237.

Paracampo, C. C. P., \& Albuquerque, L. C. (2004). Análise do papel das conseqüências programadas no seguimento de regras. Interação em Psicologia, 8(2), 237-245.

Paracampo, C. C. P., Souza, D. G., Matos, M. A., \& Albuquerque, L. C. (2001). Efeitos de mudança em contingências de reforço sobre o comportamento verbal e não verbal. Acta Comportamentalia, 9, 31-55.

Reitman, D., \& Gross, A. M. (1996). Delayed outcomes and rule-governed behavior among "noncompliant" and "compliant" boys: a replication and extension. The Analysis of Verbal Behavior, 13, 65-77.

Roberts, M. W., McMahon, R. J., Forehand, R., \& Humphreys, L. (1978). The effect of parental instructiongiving on child compliance. Behavior Therapy, 9, 793798.

Robles, H. S. M., \& Gil, M. S. C. A. (2006). O controle instrucional na brincadeira entre crianças com diferentes repertórios. Psicologia: Reflexão e Crítica, 19(2), 197205.

Romiti, R., Maragno, L., Arnone, M., \& Takahasi, M. D. F. (2009). Psoríase na infância e adolescência. Anais Brasileiros de Dermatologia, 84(1), 9-22.

Sampaio, S. A. P., \& Rivitti, E. (1998). Dermatologia (2a ed.). São Paulo: Artes Médicas.

Sanz, E. J. (2003). Concordance and children's use of medicines. British Medical Journal, 327, 858-860.

Schlinger, H., \& Blakely, E. (1987). Function-altering effects of contingency-specifying stimuli. The Behavior Analyst, 10, 41-45.

Schlinger, H. (1990). A reply to behavior analysts writing about rules and rule-governed behavior. The Analysis of Verbal Behavior, 8, 77-82.

Schmidt, A., \& De Souza, D. G. (2003). Controle instrucional: Discussões atuais. In C. E. Costa, J. C. Luzia, H. H. N. Sant'Ana (Org.). Primeiros passos em análise do comportamento e cognição (pp. 33-53). Santo André: ESETec.

Schutte, R. C., \& Hopkins, B. L. (1970). The effects of teacher attention on following instructions in a kindergarten class. Journal of Applied Behavior Analysis, 3(2), 117-122.

Sério, T. M. A. P. (2004). Comportamento verbal e o controle do comportamento humano. In T. M. A. P. Sério, M. A. Andery, P. S., Gioia, \& N. Micheletto (Org.). Controle de estímulos e comportamento operante: Uma (nova) introdução (pp. 139-164). São Paulo: EDUC.

Serup, J., Kettis, L., Åsa, M., Kjellgren, K. I., Niklasson, E., Ring, L, \& Ahlner, J. (2006). To follow or not to follow dermatological treatment - a review of the literature. Acta Dermato-Venereologica, 86(3), 193-197.

Silva, C. M. R., Gontijo, B., Pereira, L. B., \& Ribeiro, G. B. (2007). Vitiligo na infância: características clínicas e epidemiológicas. Anais Brasileiros de Dermatologia, 82(1), 47-51.

Skinner, B. F. (1966). An operant analysis of problem solving. The Behavior and Brain Sciences, 7, 283-613.

Skinner, B. F. (1969). Contingencies of Reinforcement: A Theoretical Analysis. New York: Appleton-CenturyCrofts.

Valia, R. G. (2008). Non-compliance in dermatologic diseases. Indian Journal of Dermatology, Venereology and Leprology, 74(6), 553-557. 
Wahler, R. G. (1997). On the origins of children's compliance and opposition: Family context, reinforcement, and rules. Journal of Child and Family Studies, 6, 191-208.

Zettle, R. D., \& Hayes, S. C. (1982). Rule governed behavior: A potential theoretical framework for cognitive behavior therapy. In P. C. Kendall (Ed.), Advances in cognitive behavioral research and therapy (pp. 73-118). New York: Academic.

Recebido em: 18/10/2012 Última revisão em: 29/05/2014 Aceito em: 30/06/2014

Zolnierek, K. B. H., \& DiMatteo, M. R. (2009). Physician communication and patient adherence to treatment: a meta-analysis. Medical Care, 47, 826-834.

\section{Nota:}

1 Dirigir a criança fisicamente para que ela emita comportamentos de obediência em relação à tarefa (e.g., puxá-la pela mão, buscá-la em um canto da sala, colocar o tubo de pomadas em sua mão e abrir juntamente com a criança). 